Check for updates

Cite this: RSC Adv., 2017, 7, 53525

Received 22nd August 2017

Accepted 6th November 2017

DOI: 10.1039/c7ra09283k

rsc.li/rsc-advances

\section{Controllable domain morphology in coated poly(lactic acid) films for high-efficiency and high- precision transportation of water droplet arrays $\uparrow$}

\author{
Yongtao Wang, ${ }^{a}$ Huige Yang, ${ }^{\text {a }}$ Hongzhi Liu, ${ }^{b}{ }^{b}$ Li Zhang, ${ }^{a}$ Ruixia Duan, ${ }^{a}$ \\ Xuying Liu (D) ${ }^{* a}$ and Jinzhou Chen*a
}

Construction of superhydrophobic surfaces with tunable adhesion force has attracted considerable attention in past decades. Here, we demonstrated an exfoliated surface in non-solvent-coated poly(lactic acid) (PLA) films which exhibited controllable domain morphology containing "face-on", "edge-on" or "peak-on" nano-/micro-structures. A theoretical understanding revealed that such controllable morphology responded closely to the variation in mixture viscosities at a fixed temperature. Furthermore, the resulting films were highly superhydrophobic with contact angles $>150^{\circ}$. In particular, adhesion could be tuned ranging from $144 \mu \mathrm{N}$ to $62 \mu \mathrm{N}$ for face-on and peak-on surfaces, respectively, by changing the non-solvent content. This strategy allowed for high-efficiency and high-precision transportation of water microdroplets $(1 \mu \mathrm{L})$ with a yield of $\leq 100 \%$ on homogeneous surfaces. This approach could aid no-mass-lost fluid transportation for a broad variety of applications in bioengineering and biochips.

\section{Introduction}

Superhydrophobic surfaces with tunable adhesion, such as lotus leaves and rose petals, have attracted considerable attention in recent years. ${ }^{1-3}$ The as-obtained structured surfaces with tunable adhesion enable microdroplet transportation among homogeneous surfaces (same components but with different morphologies), which can be applied widely in bio-separation, ${ }^{4}$ chemical reactions ${ }^{5}$ and patterning. ${ }^{6}$ Such surfaces must possess considerable differences in adhesion. Hence, to date, various physical methods, chemical methods, or a combination of the two, have been developed to fabricate adhesioncontrolled superhydrophobic surfaces. ${ }^{7-9}$

Interestingly, superhydrophobicity has been observed in porous surfaces with nano-/micro-structures in polymer films. ${ }^{10-14}$ Porosity formation is caused by the instability of a polymer in bicontinuous fluid mixtures, which induces the spinodal decomposition, and blooms further into nano-phase separation (NPS). ${ }^{15}$ In particular, if a miscible solvent/nonsolvent mixture is used, the NPS at the initial stage yields a solute-rich phase and a solute-poor phase. Ultimately, the former gives rise to nano-/micro-structure-containing domains,

${ }^{a}$ School of Materials Science and Engineering, Zhengzhou University, Zhengzhou 450001,China.E-mail: cjz@zzu.edu.cn; yanghg@zzu.edu.cn; liuxy@zzu.edu.cn ${ }^{b}$ College of Engineering, Zhejiang Agriculture \& Forestry University, Hangzhou, 311300, China

$\dagger$ Electronic supplementary information (ESI) available. See DOI: $10.1039 / \mathrm{c} 7 \mathrm{ra} 09283 \mathrm{k}$ whereas the latter forms hierarchically nano-/micro-scale pores. Furthermore, superhydrophobicity in the porous surface can be interpreted using the Cassie eqn (1): ${ }^{16}$

$$
\cos \theta_{\mathrm{c}}=f_{1} \cos \theta-f_{2}
$$

where $\theta_{\mathrm{c}}$ and $\theta$ are the contact angles (CAs) of the porecontaining surface and nonporous surface, respectively. $f_{1}$ is the fraction of the solid surface in contact with water and $f_{2}$ is the fraction of air in contact with water $\left(f_{1}+f_{2}=1\right)$. Apparently, increasing the volume ratio of the non-solvent : solvent enables a decrease in the fraction $\left(f_{1}\right)$ of liquid-solid contact, thereby making the surface superhydrophobic with a large value of $\theta_{\mathrm{c}}$. However, according to a report from Wenzel, ${ }^{17}$ the surface adhesion $(F)$ can be defined as a function of the contact fraction $\left(f_{1}\right)$ between solid surfaces and the water droplet. The adhesion force can be expressed by eqn (2):

$$
F=k I f_{1}
$$

where $k$ is a constant and $I$ is an indicator of the solid surface chemistry, thereby indicating that $F$ increases in proportion to $f_{1}$. In this case, a high adhesion $F$ value calls for a large $f_{1}$. However, large $f_{1}$ values inhibit the increase in the CA $\theta_{c}$, as indicated in eqn (1), thus lowering the superhydrophobicity. Therefore, taking account of how to gain tunable adhesion in a superhydrophobic surface, how $f_{1}$ can be adjusted using surface morphology becomes very important. Jiang et al. reported that the preparation of porous superhydrophobic 
surfaces using an electrochemical anodizing method enabled tuning of the adhesion force of water droplets. ${ }^{18}$ Since then, a wide variety of methods, including femtosecond laser weaving, ${ }^{19}$ spray-coating of heterogeneous chemical compositions $^{20}$ and stereo-complex co-crystallization, ${ }^{21}$ have been developed to realize a tunable adhesive superhydrophobic surface with heterogeneous chemical compositions. Inevitably, most of these methods suffer from the use of expensive lowsurface-energy components (e.g. fluorides and silanes) or complicated fabrication procedures for obtaining appropriate surface roughness, which limit the applications of superhydrophobic surfaces.

Here, we chose a bio-compatible polymer of poly(lactic acid) (PLA) as our model. PLA is a crucial biodegradable material in bio-medical areas, so creating a superhydrophobic PLA framework surface very important. In this sense, an exfoliation method was developed first in coated PLA films to fabricate the superhydrophobic surface with adhesion-tuning force over a wide range and gave a theoretical understanding for the dynamics of crystal growth. On the surface, the exfoliating process could remove the crystal surface grown initially slightly. This process revealed the inner hierarchically microporous structures with controlled-domain morphology, thereby yielding a superhydrophobic and tunable adhesion thin film due to the different contact areas with water droplets. In particular, when the volume ratio of the non-solvent : solvent was changed from $50 \%$ to $90 \%$, the obtained surfaces exhibited high superhydrophobicity with a CA of $>150^{\circ}$ and a wide variation of water adhesive force ranging from $144 \mu \mathrm{N}$ to $62 \mu \mathrm{N}$ for "face-on", "edge-on" and "peak-on" orientations. These surfaces allow for high throughput to transport water microdroplets ( $1 \mu \mathrm{L}$, even $96 \%$ in a $7 \times 7$ array) on the scaling-up surface. This strategy could be used for the efficient no-mass-lost transportation of microdroplets in congener materials for biological applications such as cell cultures, bioengineered tissues and biochips.

\section{Materials and methods}

\subsection{Sample preparation}

PLA substrates of dimension $50 \mathrm{~mm} \times 10 \mathrm{~mm} \times 2 \mathrm{~mm}$ were prepared using an injection-molding machine (F80WZ, Ningbo Haitian, China), and the temperature was set at $180{ }^{\circ} \mathrm{C}, 175^{\circ} \mathrm{C}$, $180{ }^{\circ} \mathrm{C}$ and $180^{\circ} \mathrm{C}$.

PLA coatings were fabricated using a non-solvent-induced phase-separation method according to a procedure reported previously. ${ }^{22}$ In this work, chloroform rather than dichloromethane was chosen as a solvent for PLA, whereas $n$-butyl acetate, absolute ethyl alcohol and $n$-butyl alcohol were mixed together at a volume ratio of $1: 1: 1$ and used as a ternary nonsolvent. First, $1.2 \mathrm{~g}$ PLA was dissolved in $24 \mathrm{~mL}$ chloroform under magnetic stirring to form a homogeneous solution. Then, the ternary non-solvent was added into an equivalent PLA solution to form a homogeneous mixture under stirring. The volume ratio of the non-solvent : solvent was varied $(50 \%, 60 \%$, $70 \%, 80 \%$, and $90 \%$ ).

Superhydrophobic PLA surfaces with water tunable adhesion were fabricated using a new coating-exfoliated method.
Specifically, the PLA solution $(1.5 \mathrm{~mL})$ in the chloroform/nonsolvent mixture was coated onto the PLA substrate using a drop-coating method with a burette to form a superhydrophobic PLA layer. After drying at ambient temperature, the resulting surface was peeled-off with tapes under $5 \pm 1 \mathrm{~N}$, and fresh PLA surfaces with different crystal orientations were obtained.

\subsection{No-mass-loss transportation of droplet array}

Transport photographs of droplet $(10 \mu \mathrm{L})$ array in a $40 \mathrm{~mm} \times$ $40 \mathrm{~mm}$ PLA substrate from low adhesion (PLA-5; the ratio of non-solvent : solvent $=90 \%$ ) to high adhesion (PLA-1; the ratio of non-solvent : solvent $=50 \%$ ) were taken using a camera (DSC-H400; Sony) and the position of both substrates was constant $(2 \mathrm{~mm})$. The transport efficiency was calculated using the following formula:

\section{Transport efficiency $(\%)=$ number of transported droplets/ number of total droplets $\times 100 \%$}

\subsection{Mechanical properties}

The mechanical features of the various PLA substrates were measured using a universal tensile testing machine (CMT6104; Suns, China) at room temperature. Tensile strength was measured according to specification GB/T 1040.2-2006 (ISO 527$2: 1993)$. The extension rate was $2 \mathrm{~mm} \mathrm{~min}^{-1}$ and the load cell was $10 \mathrm{kN}$. The PLA substrate was a standard form $(200 \mathrm{~mm} \times$ $10 \mathrm{~mm} \times 4 \mathrm{~mm})$. Bending strength and impact strength measured through GB/T 9341-2008 (ISO 178:2001) and GB/T 1843-2008 (ISO 180:2000), respectively. Five samples of each PLA substrate were tested and average values obtained.

\section{Results and discussion}

\subsection{Exfoliation process in coated PLA films}

Fig. 1a shows the evolution of phase separation in a binary mixture of solvent and non-solvent. A non-solvent mixture with gradient viscosities was used to control phase separation in a more moderate manner than that of a single solution. With increasing phase separation, the solution started to form two phases: a solute-rich phase and a solute-poor phase. Finally, after the completion of phase separation and complete drying up of the solvent, nano-/micro-structures could be observed in solute-rich phase regions when the upper surface was removed, but was observed rarely in the solute-rich phase regions. According to this mechanism, we carried out an experiment using a bio-compatible polymer of PLA as the solute, chloroform as the solvent, and an alcohol mixture as the non-solvent as described in the Materials and methods section.

The PLA solution was deposited onto the pristine PLA substrates to obtain the desired coatings (Fig. 1b). Here, other substrates, such as glass and silica, were also used, but exhibited poor adhesion to the deposited PLA, which did not perform as well as the pristine PLA surface. PLA crystals started to grow as the solvent evaporated. After the solvent had 
a
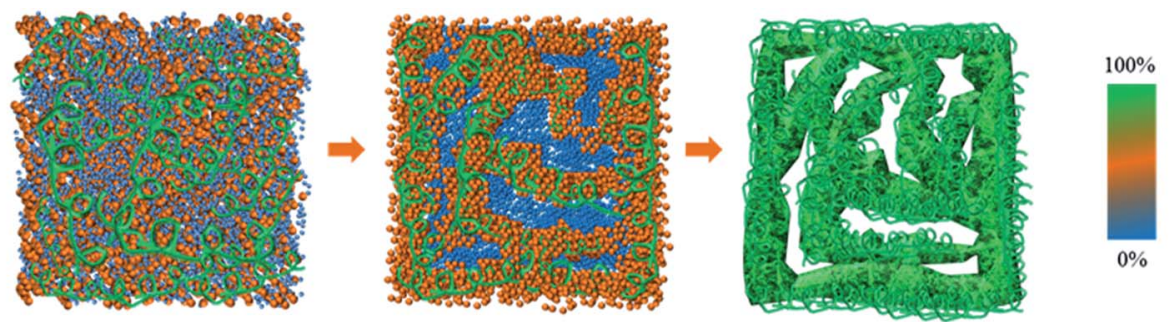

$6^{6}$ PLA Molecule

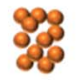

Solvent Phase

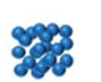

Non-Solvent Phase
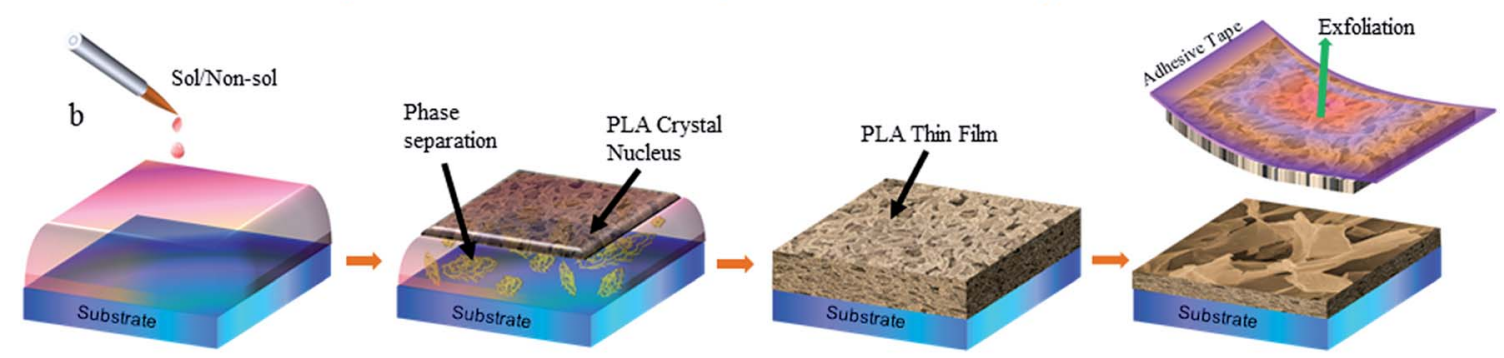

Fig. 1 (a) Mapping of microporous PLA structures originating from the phase-separation process (schematic). The coloured bar graph on the right represents the PLA concentration. (b) Strategy for fabricating superhydrophobic surfaces with tunable adhesion (schematic).

evaporated completely, an adhesive tape was used to remove the top surface to unveil the inner crystal domains. There were two types of crystal-growth processes initially. At the interface between the liquid film and air, PLA crystals grew rapidly due to the rapid evaporation rate of the solvent and non-solvent. This led to a rough surface with uncontrolled-growth nano-/microstructures, which showed a hydrophobic property, ${ }^{23}$ but nontuned adhesion forces (Fig. S1 of ESI†). Crystal growth in the bulky region of the liquid film could be adjusted delicately by the interaction between the inner solvent and non-solvent.

\subsection{Controllable domain morphology}

Fig. 2 shows the scanning electron microscope (SEM) images and illustration of selected samples from all of nano-/micro- structures-containing surfaces. When phase separation was not carried out, the pristine PLA surface showed only a few nanostructures (Fig. 2a and e), whereas the PLA surfaces with nanophase separation had vary abundant and diverse nano-/ micro-structures. In particular, these structures were strongly dependent on the non-solvent volume fraction. When a low fraction $(<60 \%)$ was used in this system, lance-shaped structures could be formed, which stacked together in a parallel fashion with a face-on configuration (Fig. $2 b$ and f). Increasing the ratio of the non-solvent volume resulted in a significant change in surface morphology. A moderately high ratio of $70 \%$ yielded quite fine structures with an edge-on configuration (Fig. $2 \mathrm{c}$ and g), which appeared as narrow spiral lines. When the ratio reached a high value (>90\%), the nano-structures looked

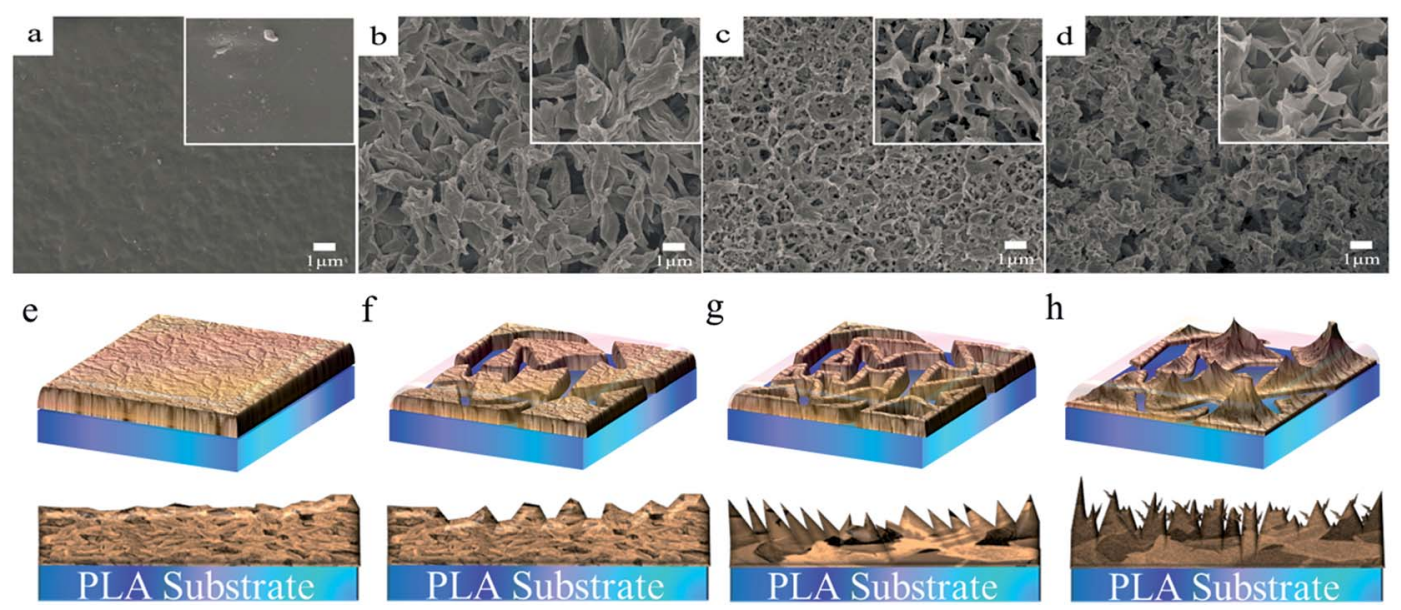

Fig. 2 SEM images of a pristine PLA surface (a) and exfoliated film surfaces with controllable domain morphology (b)-(d). The non-solvent rates are $60 \%, 70 \%$, and $90 \%$ for (b), (c) and (d), respectively. The insets show magnified zones ( $\times 3$ ). Nano-/micro-structures (top view and crosssection) in PLA surfaces (e)-(h) corresponding to the SEM images shown above from (a) to (d), respectively (schematic). 
like discontinuous "mountain peaks" (Fig. $2 \mathrm{~d}$ and $\mathrm{h}$ ). In addition, different ternary systems, such as a PLA-chloroformalcohol system, were also used to fabricate superhydrophobic surfaces using this exfoliation process, and showed adhesioncontrolled behaviour, as mentioned above. These results indicated that the exfoliation process was a common method to obtain superhydrophobic surfaces.

\subsection{Theoretical comprehension}

To gain a better understanding of the controllable growth mechanism of such nano-/micro-structures originating from non-solvent-volume-ratio-dependent nanophase separation, we resorted to the theory of spinodal decomposition in the solvent/ non-solvent mixture developed by Binder et al. ${ }^{24}$ For an asymmetric composition (especially for a non-solvent volume ratio $>0.5$ ), the nano-/micro-structure growth is driven by the hydrodynamic diffusion process of the solvent droplet. Hence, the mean size of a nano-/micro-structure or the inter distance $R$ can be estimated in a straightforward fashion by eqn (3) and (4):

$$
\begin{gathered}
R^{2} \sim D_{\mathrm{R}} t \\
D_{\mathrm{R}} \sim k_{\mathrm{B}} T /(5 \pi \eta R)
\end{gathered}
$$

where $t$ is the phase separation time, $D_{\mathrm{R}}$ is the diffusion factor, $k_{\mathrm{B}}$ is Boltzmann's constant, $T$ is the temperature and $\eta$ is the mixture viscosity. Then, we can extract the growth law for the PLA domain using eqn (5):

$$
R \sim\left(\kappa_{\mathrm{B}} T / \eta\right)^{1 / 3} t^{1 / 3}
$$

Therefore, at a fixed temperature, the area of the nano- $/$ micro-structure is proportional to the processing time, but inversely proportional to the viscosity. In this mixture of solvent and non-solvent, if the interaction between components is neglected, the viscosity can, in general, be assumed according to the following relationship (6): ${ }^{25}$

$$
\ln \eta=X_{1} \ln \eta_{1}+X_{2} \ln \eta_{2}
$$

where $X_{1}, X_{2}, \eta_{1}$, and $\eta_{2}$ are the mole fractions and viscosities of solvent and non-solvent used in the mixture. As mentioned in the Materials and methods section, chloroform was chosen as a solvent $(\eta=0.56)$. A mixture of $n$-butyl acetate, absolute ethyl alcohol and $n$-butyl alcohol at a volume ratio of $1: 1: 1$ was used as non-solvent $(\eta=1.09)$. A monotonic increase or decrease in the mixture viscosity could be predicted as the nonsolvent volume fraction increased. Thus, as the high-viscosity non-solvent volume ratio increased, the solution viscosity increased simultaneously (Fig. S1 of ESI $\dagger$ ). This led to a decrease in the size of the nano-/micro-structure (Fig. S2 of ESI $\dagger$ ) by taking eqn (5) and (6) into account, which was not consistent with the observation shown in Fig. 2.

\subsection{CA and sliding angle (SA) of water}

These nano-/micro-structure-containing PLA surfaces exhibited quite different interactions with water droplets. When the water droplets were placed statically on the resulting PLA surfaces, the CAs were significantly high even up to $155^{\circ}$, and showed a slight increasing trend as the non-solvent volume fraction increased (Fig. 3a), whereas the as-coated PLA surfaces did not satisfy the requirement of superhydrophobicity. The type of sliding of water droplets on these surfaces was different for each water droplet (Fig. 3b) and negatively followed the increase in nonsolvent content. The tunable CA value and SA were closely related to the finely controlled surface morphology because the pristine PLA surface showed a CA of just $72^{\circ} .{ }^{26}$ The difference in the CA and SA detected in the obtained PLA surfaces was ascribed mainly to the area variation in the nano-/microstructures (Fig. 3c-e). For example, the very fine nanostructures illustrated in Fig. 3e yielded the weakest interaction with a water droplet ever reported, ${ }^{27}$ and thus formed a smaller contact area, leading to the highest CA and lowest SA. A further understanding can be made by combining the SEM images in Fig. 2 to produce the schematic illustration in Fig. 3f. The faceon configuration has the largest solid-liquid contact area, thus giving rise to the lowest CA and highest SA values, whereas the point-on configuration presents converse behaviour. The reason for these phenomena lies in the fact that three phase contact lines (air phase, liquid phase, and solid phase) have important roles in controlling the adhesion force, rather than the difference in CAs. That is, the SA depends on the type of contact, whereas the CA depends on micro-/nanoscale morphology. Besides, smaller CAs and higher SAs can be achieved on the surfaces fabricated at an increased temperature (Fig. S3 of ESI $\dagger$ ) because the surface areas of nano-structures increase upon heating. Therefore, the experimental results and theoretical understanding were in agreement.

\subsection{Measurement of dynamic adhesion}

The measurement of dynamic adhesion was carried out to investigate the further tuning of the surface interaction between the fabricated nano-/micro-structures and water droplets. Delicately control of adhesion behaviours was observed (Fig. 4a and b). In particular, the adhesive forces decreased gradually with an increase in the non-solvent, which was similar to the variation observed in SAs. For a volume ratio of $50 \%$, the adhesive force was $\leq 144 \mu \mathrm{N}$, which is comparable with that of a rose petal $(140 \mu \mathrm{N}) .^{28}$ The adhesive forces of other samples with volume ratios from $60 \%$ to $90 \%$ ranged from $121 \mu \mathrm{N}$ to $62 \mu \mathrm{N}$ (Fig. 4c). Importantly, the nearly linear trend further confirmed that the tuning of adhesive force could be ascribed to varying the non-solvent volume fraction, which resulted in a gradual increase in the solution viscosity (Fig. 4d). As a comparison, the water adhesive force of pristine PLA was $>200 \mu \mathrm{N}$, which could be ascribed mainly to the smooth surface and lower CA. This variation in the water adhesion originated primarily from the surface micro-/nanostructures, as discussed above. Thus, superhydrophobic surfaces with adhesion that could be tuned continuously from a high state to a low state had been fabricated. These were homogeneous PLA films with slightly different crystallinity and mechanical properties (Fig. S4, Tables S2 and S3 of ESI $\dagger$ ). 

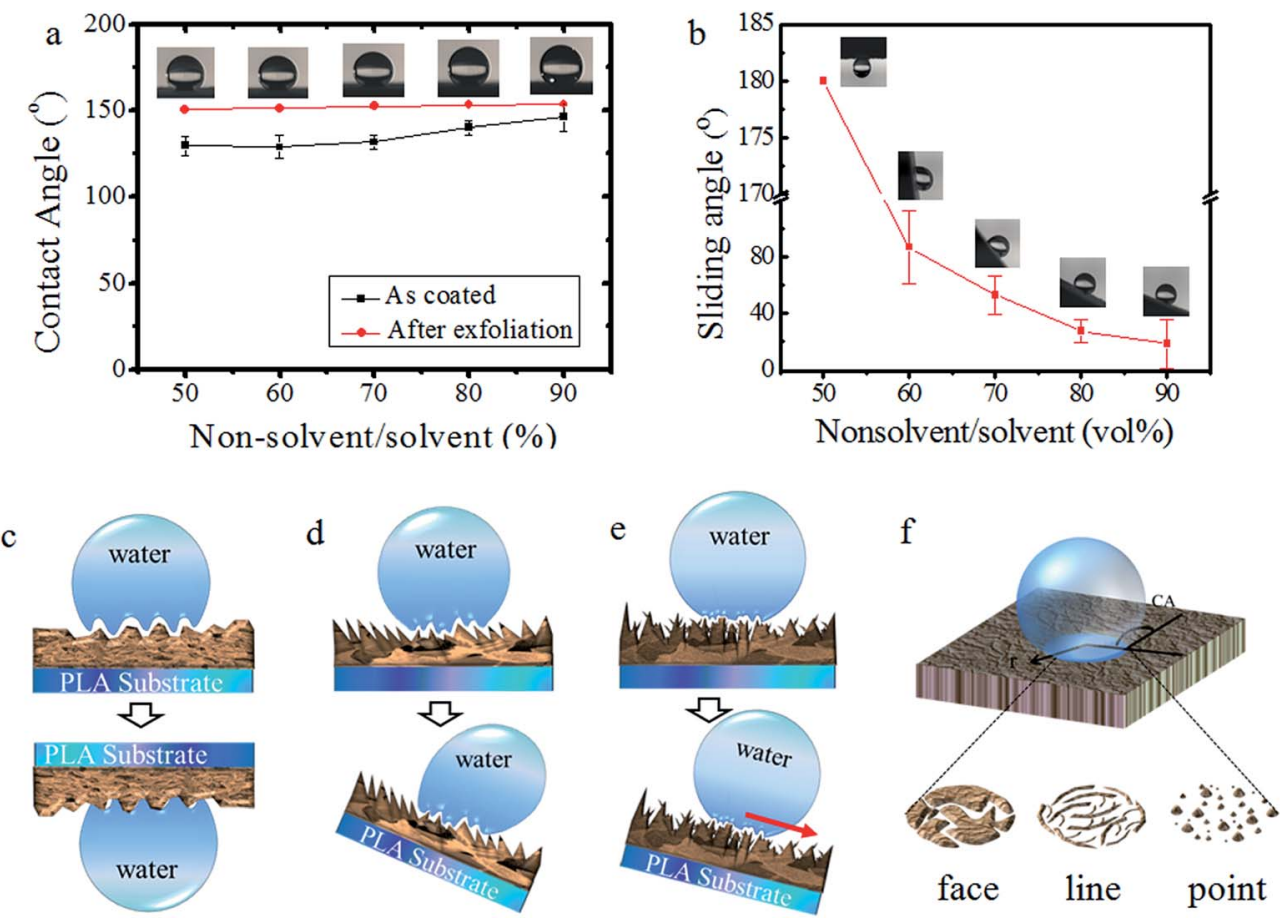

Fig. 3 (a) and (b) The dependence of the contact angle and sliding angle of water on the volume ratio of the non-solvent : solvent, respectively. These insets are photographs of contact angles and sliding angles on different PLA surfaces. Schematic illustrations of the three models of superhydrophobic surfaces with an adhesive force ranging from high to low: (c) area-contact type, (d) line-contact type, and (e) point-contact type (the red arrow represents the roll-down direction). (f) Three solid-liquid contact types: face, line and point, respectively.

\subsection{No-mass-loss transporting for micro-droplet arrays}

Practical applications could be demonstrated by applying the obtained superhydrophobic PLA surfaces in a no-mass-loss transportation platform for micro-droplet arrays (Fig. 5). First, a schematic illustration of droplet-array transportation from low adhesion to high adhesion is displayed in Fig. 5a. A $5 \times 5$ array of water droplets $(10 \mu \mathrm{L})$ was placed statically on the PLA-5 surface (ratio of non-solvent : solvent $=90 \%$ ) with low adhesion

\section{a}
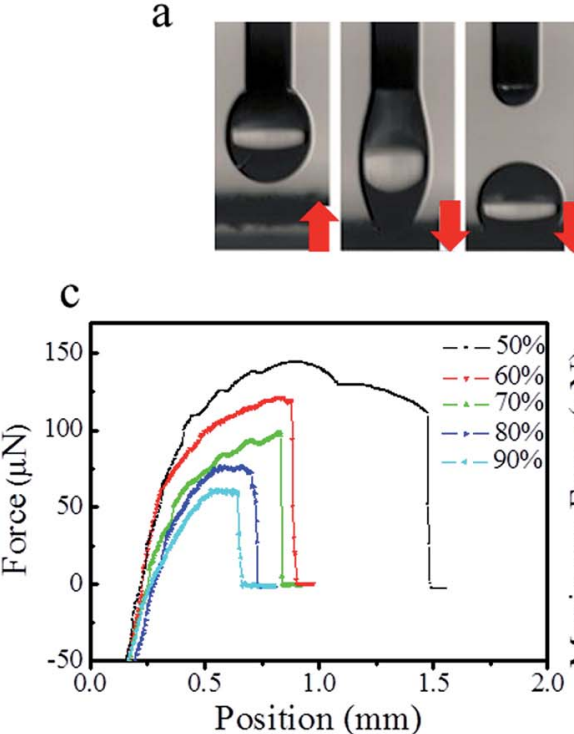

$\mathrm{b}$

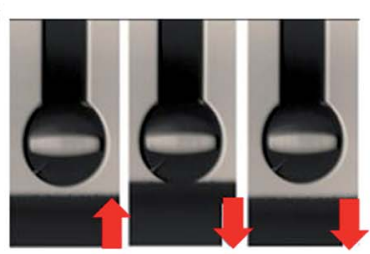

$\mathrm{d}$

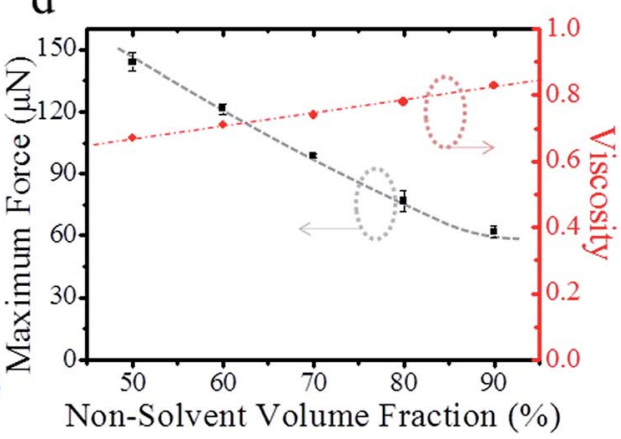

Fig. 4 (a) and (b) The profiles of a water droplet ( $5 \mu \mathrm{L}$ ) on superhydrophobic surfaces (top) and the interaction between the droplet and the PLA surface through an up-and-down platform, $4 \mathrm{~mm} \mathrm{~min}^{-1}$ (volume ratio, 50\% and $90 \%$, respectively; the red arrows represent the moving directions of the stages). (c) Real-time recorded force-distance curves for a series of PLA surfaces during the measurement of dynamic adhesion. (d) The dependence of the non-solvent volume fraction on maximum force and viscosity at room temperature. 
a

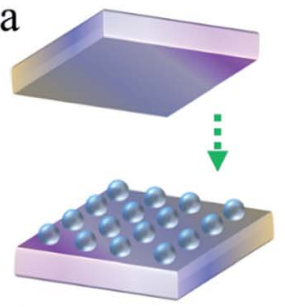

c

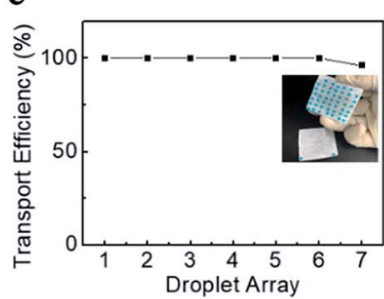

15

f

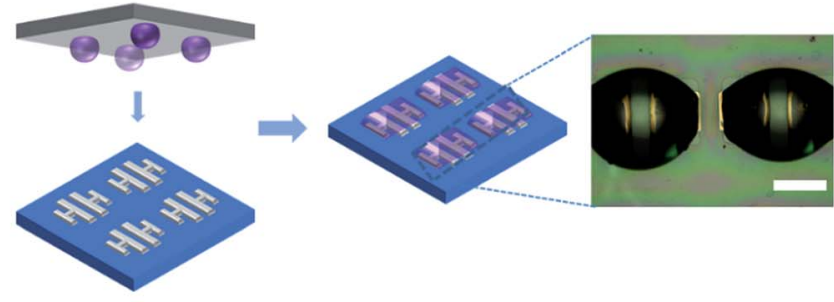

d

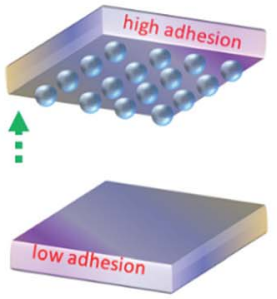

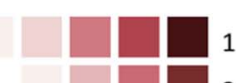

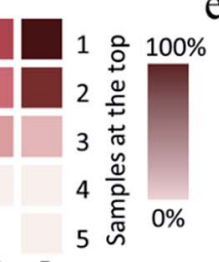

b

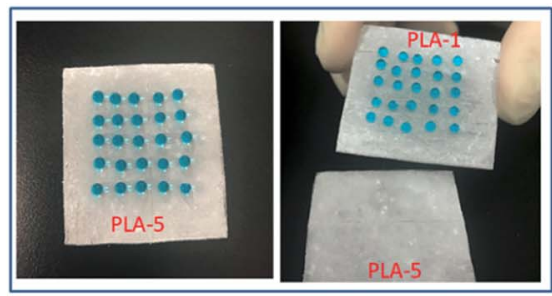

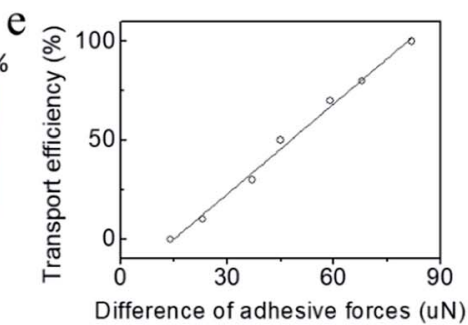

g

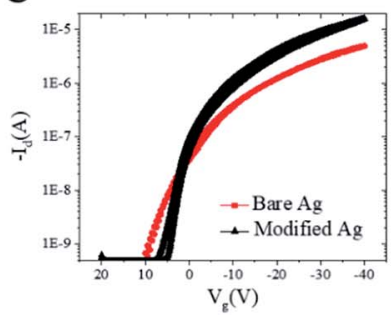

Fig. 5 No-mass-loss transportation of water droplet arrays. (a) Illustration of droplet-array transportation; (b) transport photographs of a $5 \times 5$ droplet $(10 \mu \mathrm{L})$ array in a PLA substrate $(40 \mathrm{~mm} \times 40 \mathrm{~mm}$ ) from PLA-5 (ratio of non-solvent : solvent $=90 \%)$ to PLA-1 (ratio of non-solvent : solvent $=50 \%)$ when the position of both substrates is constant $(2 \mathrm{~mm})$; (c) the effect of droplet-array number and density on transport efficiency; (d) the relationship between various PLA surfaces and the transport efficiency of water droplets; the numbers 1, 2, 3, 4, and 5 represent the different PLA surfaces (ratio of non-solvent : solvent $=50,60,70,80$, and $90 \%$, respectively); (e) transport efficiency is a function of the difference in adhesive forces and $R^{2}=0.993$. (f) High-precision transport of conductive aqueous ink-droplet arrays ( $25 \mathrm{droplets,} 1 \mu \mathrm{L}$ ) in solutionpatterned electronics (scale bar $=200 \mu \mathrm{m}$ ). (g) Device performance.

(Fig. 5b and ESI Movie S1 $\dagger$ ), which could be transferred readily by moving the PLA-1 surface (ratio of non-solvent : solvent $=$ $50 \%)$ with super-high adhesion. Moreover, the droplet array could be transported completely from low-adhesion surfaces to high-adhesion surfaces without any mass loss.

The effect of droplet-array number and density on transport efficiency was investigated (Fig. 5c). The droplet array could be fully transferred from PLA-5 to PLA-1 even with a high-density array of water droplets, which is usually required in microreactors. ${ }^{29}$ When an array of $7 \times 7$ droplets was implemented, two droplets at the edge failed to be transported, probably owing to the defects along the edge regions that did not exhibit the microstructures observed in the central regions. Fig. 5d shows the relationship between various PLA surfaces and transport efficiency, and indicated that a greater difference between adhesive forces led to the higher transport efficiency observed in Fig. 5e. In addition, this approach is suitable for the transport of micro-scale aqueous conductive ink droplets for high-resolution patterning of electronics to fabricate biochips at room temperature. ${ }^{30,31} \mathrm{~A} 5 \times 5$ array with a droplet volume of $1 \mu \mathrm{L}$ was transported by the PLA surface to the device array (Fig. 5f). After modification using this method, the device performance was enhanced significantly with positive carrier mobility of $>2 \mathrm{~cm}^{2} \mathrm{~V}^{-1} \mathrm{~s}^{-1}$, which is nearly fivefold higher than that in non-modified arrays (Fig. 5g). This high-throughput fluid transport indicates that the fabricated superhydrophobic PLA surfaces with high adhesion could be applied in biological assays such as cell cultures. ${ }^{32}$

\section{Conclusion}

In the present study, a series of superhydrophobic PLA surfaces with tunable adhesion for water were unveiled through an exfoliated method in non-solvent-coated PLA films. The obtained surfaces exhibited controllable domain morphology, which allowed the existence of three contact types: face contact, edge contact and peak contact. A hypothesis was proposed to understand the formation mechanism of such controllable morphology: close dependence on viscosity variation in the mixture at a fixed temperature. Furthermore, by increasing the volume ratio of non-solvent: solvent from $50 \%$ to $90 \%$, the adhesive force of water decreased from $144 \mu \mathrm{N}$ to $62 \mu \mathrm{N}$, and SAs could be adjusted from a high value to a low one. The underlying mechanism for such behaviour was attributed to the different states of superhydrophobic surfaces. Moreover, the proposed method will aid understanding of superhydrophobicity and the 
design of homogeneous surfaces with tunable adhesion. Besides, demonstration of application of these PLA surfaces in no-massloss transport of large-area water-droplet arrays reveals their promising potential in biochips.

\section{Conflicts of interest}

There are no conflicts to declare.

\section{Acknowledgements}

We gratefully acknowledge the financial support from the key research project plan of Higher Learning Institutions of Henan Province (17B430008), the National Natural Science Foundation of China (51573169 and 61704155), and the Startup Research Fund for Young teachers of Zhengzhou University (F0000907).

\section{References}

1 B. Su, Y. Tian and L. Jiang, J. Am. Chem. Soc., 2016, 138, 1727.

2 W. F. Zhang, X. Lu, Z. Xin and C. L. Zhou, RSC Adv., 2016, 6, 106054.

3 T. Zhu, C. Cai, J. Guo, R. Wang, N. Zhao and J. Xu, ACS Appl. Mater. Interfaces, 2017, 9, 10224.

4 S. Ali, W. Tian, N. Ali, L. X. Shi, J. Kong and N. Ali, RSC Adv., 2015, 5, 7160.

5 S. Shin, J. Seo, H. Han, S. Kang, H. Kim and T. Lee, Materials, 2016, 9, 116.

6 M. Liu and L. Jiang, Adv. Funct. Mater., 2010, 20, 3753.

7 J. Li, X. Liu, Y. Ye, H. Zhou and J. Chen, J. Phys. Chem. C, 2011, 115, 4726.

8 B. N. Sahoo and B. Kandasubramanian, RSC Adv., 2014, 4, 22053.

9 M. Toma, G. Loget and R. M. Corn, ACS Appl. Mater. Interfaces, 2014, 6, 11110.

10 H. Y. Erbil, A. L. Demirel, Y. Avcı and O. Mert, Science, 2003, 299, 1377.

11 M. He, Q. Zhang, X. Zeng, D. Cui, J. Chen, H. Li, J. Wang and Y. Song, Adv. Mater., 2013, 25, 2291.

12 Y. Li, S. Chen, M. Wu and J. Sun, Adv. Mater., 2014, 26(20), 3344.
13 N. Xu, D. K. Sarkar, X. G. Chen, H. Zhang and W. P. Tong, RSC Adv., 2016, 6, 35466.

14 Z. Xue, Y. Cao, N. Liu, L. Feng and L. Jiang, J. Mater. Chem. A, 2014, 2, 2445.

15 P. Adelhelm, Y. S. Hu, L. Chuenchom, M. Antonietti, B. M. Smarsly and J. Maier, Adv. Mater., 2007, 19, 4012.

16 M. Miwa, A. Nakajima, A. Fujishima, K. Hashimoto and T. Watanabe, Langmuir, 2000, 16, 5754.

17 R. N. Wenzel, Ind. Eng. Chem., 1936, 28, 988.

18 Y. Lai, X. Gao, H. Zhuang, J. Huang, C. Lin and L. Jiang, Adv. Mater., 2009, 21, 3799.

19 D. Zhang, F. Chen, Q. Yang, J. Yong, H. Bian, Y. Ou, J. Si, X. Meng and X. Hou, ACS Appl. Mater. Interfaces, 2012, 4, 4905.

20 J. Li, Z. Jing, F. Zha, Y. Yang, Q. Wang and Z. Lei, ACS Appl. Mater. Interfaces, 2014, 6, 8868.

21 A. Gao, Y. Zhao, Q. Yang, Y. Fu and L. Xue, J. Mater. Chem. A, 2016, 4, 12058.

22 Y. Chang, X. Liu, H. Yang, L. Zhang, Z. Cui, M. Niu, H. Liu and J. Chen, Soft Matter, 2016, 12, 2766.

23 P. X. Ma, Mater. Today, 2004, 7, 30.

24 K. Binder and D. Stauffer, Phys. Rev. Lett., 1974, 33, 1006.

25 A. K. Doolittle, J. Appl. Phys., 1951, 22, 1471.

26 A. Ortner, A. Pellis, C. Gamerith, A. O. Yebra, D. Scaini, I. Kaluzna, D. Mink, S. de Wildeman, E. H. Acero and G. M. Guebitz, RSC Adv., 2017, 19, 816.

27 H. Y. Guo, Q. Y. Li, H. P. Zhao, K. Zhou and X. Q. Feng, RSC $A d v .$, 2015, 5, 66901.

28 F. Lin, Y. Zhang, J. Xi, Y. Zhu, N. Wang, F. Xia and L. Jiang, Langmuir, 2008, 24, 4114.

29 L. H. Hung and A. P. Lee, J. Med. Bioeng., 2007, 27, 1.

30 X. Liu, M. Kanehara, C. Liu, K. Sakamoto, T. Yasuda, J. Takeya and T. Minari, Adv. Mater., 2016, 28, 6568.

31 X. Liu, C. Liu, K. Sakamoto, T. Yasuda, P. Xiong, L. Liang, T. Yang, M. Kanehara, J. Takeya and T. Minari, NPG Asia Mater., 2017, 9, e409.

32 X. Hong, X. Gao and L. Jiang, J. Am. Chem. Soc., 2007, 129, 1478. 